\title{
Testing hadronic interaction models with the attenuation length of muons in KASCADE-Grande
}

\author{
J.C. Arteaga-Velázquez ${ }^{* 1}$, W.D. Apel ${ }^{2}$, K. Bekk ${ }^{2}$, M. Bertaina ${ }^{3}$, J. Blümer ${ }^{2,4}$, H. Bozdog ${ }^{2}$, \\ I.M. Brancus ${ }^{5}$, E. Cantoni ${ }^{3,6}$, A. Chiavassa ${ }^{3}$, F. Cossavella ${ }^{4}$, K. Daumiller ${ }^{2}$, V. de Souza ${ }^{7}$, \\ F. Di Pierro ${ }^{3}$, P. Doll ${ }^{2}$, R. Engel ${ }^{2}$, D. Fuhrmann ${ }^{8}$, A. Gherghel-Lascu ${ }^{5}$, H.J. Gils ${ }^{2}$, \\ R. Glasstetter ${ }^{8}$, C. Grupen ${ }^{9}$, A. Haungs ${ }^{2}$, D. Heck ${ }^{2}$, J.R. Hörandel ${ }^{20}$, D. Huber ${ }^{4}$, T. Huege ${ }^{2}$, \\ K.-H. Kampert ${ }^{8}$, D. Kang ${ }^{4}$, H.O. Klages ${ }^{2}$, K. Link ${ }^{4}$, P. Łuczak ${ }^{11}$, H.J. Mathes ${ }^{2}$, H.J. Mayer ${ }^{2}$, \\ J. Milke ${ }^{2}$, B. Mitrica ${ }^{5}$, C. Morello ${ }^{6}$, J. Oehlschläger ${ }^{2}$, S. Ostapchenko ${ }^{12}$, N. Palmieri ${ }^{4}$, \\ T. Pierog ${ }^{2}$, H. Rebel ${ }^{2}$, M. Roth ${ }^{2}$, H. Schieler ${ }^{2}$, S. Schoo ${ }^{2}$, F.G. Schröder ${ }^{2}$, O. Sima ${ }^{13}$, \\ G. Toma ${ }^{5}$, G.C. Trinchero ${ }^{6}$, H. Ulrich ${ }^{2}$, A. Weindl ${ }^{2}$, J. Wochele ${ }^{2}$, J. Zabierowski ${ }^{11}$ - \\ KASCADE-Grande Collaboration \\ ${ }^{1}$ Instituto de Física y Matemáticas, Universidad Michoacana, Morelia, Mexico \\ ${ }^{2}$ Institut für Kernphysik, KIT - Karlsruhe Institute of Technology, Germany \\ ${ }^{3}$ Dipartimento di Fisica, Università degli Studi di Torino, Italy \\ ${ }^{4}$ Institut für Experimentelle Kernphysik, KIT - Karlsruhe Institute of Technology, Germany \\ ${ }^{5}$ Horia Hulubei National Institute of Physics and Nuclear Engineering, Bucharest, Romania \\ ${ }^{6}$ Osservatorio Astrofisico di Torino, INAF Torino, Italy \\ ${ }^{7}$ Universidade São Paulo, Instituto de Física de São Carlos, Brasil \\ ${ }^{8}$ Fachbereich Physik, Universität Wuppertal, Germany \\ ${ }^{9}$ Department of Physics, Siegen University, Germany \\ ${ }^{10}$ Dept. of Astrophysics, Radboud University Nijmegen, The Netherlands \\ ${ }^{11}$ National Centre for Nuclear Research, Department of Astrophysics, Lodz, Poland \\ ${ }^{12}$ Frankfurt Institute for Advanced Studies (FIAS), Frankfurt am Main, Germany \\ ${ }^{13}$ Department of Physics, University of Bucharest, Bucharest, Romania \\ E-mail: arteaga@ifm.umich.mx
}

Preliminary analyses of air-shower data from the KASCADE-Grande observatory have pointed out a possible discrepancy between the predicted and the measured values of the attenuation length of muons with energy threshold of $230 \mathrm{MeV}$ at ground level in air showers. In particular, the analyses suggest that the measured muon attenuation length, as reconstructed with the constant intensity cut method, could be larger than the expected values from the QGSJET-II-2, QGSJET-II04, SIBYLL 2.1 and EPOS 1.99 hadronic interaction models for showers with energies between $10^{16.3}$ and $10^{17.3} \mathrm{eV}$. In this contribution, we investigate the aforementioned anomaly using a more detailed analysis than in previous works. The study involves the identification and the calculation of the most relevant systematic uncertainties affecting both measurements and simulations. From the results of this analysis, we show that the predictions from the modern high-energy hadronic interaction models on the muon attenuation length are not statistically consistent with the measured value at KASCADE-Grande.

The 34th International Cosmic Ray Conference,

30 July- 6 August, 2015

The Hague, The Netherlands 


\section{Introduction}

At high energies, cosmic ray studies rely on the measurements and analyses of the extensive air showers (EAS) that cosmic rays induce in the atmosphere. However, the interpretation of the data is not an easy task as it is hampered by the uncertainties of the high-energy hadronic interaction models. Although these uncertainties have been reduced with the arrival of the LHC, post-LHC models still exhibit some deficiencies when describing the EAS data. For example, preliminary studies performed on the KASCADE-Grande air-shower data $[1,2,3]$ have pointed out the existence of a possible discrepancy between the measured zenith angle dependence of the muon content of EAS at the observatory level and the predictions of the post-LHC models QGSJET-II-04 [4] and EPOS-LHC [5]. In particular, the above studies indicate that the attenuation length of the muon content of EAS in the atmosphere in the energy interval from $10^{16.3}$ to $10^{17.3} \mathrm{eV}$ is larger than the one predicted not only by the new hadronic interaction models compatible with the LHC, but also by the pre-LHC models QGSJET-II-2 [6], EPOS 1.99 [7] and SIBYLL 2.1 [8]. Muons are the direct messengers of the hadronic processes occurring in the shower. Therefore a failure of the models to describe the observed muon data of EAS may imply a problem with the current hadronic interaction models. In this regard, the abovementioned anomaly has received an important attention at KASCADE-Grande.

The first question to be answered is whether the muon discrepancy that has been observed in KASCADE-Grande is caused by a systematic/statistical effect. Then, if systematic effects are ruled out, what is the significance of the results and to which extend the predictions of simulations are not in agreement with the experimental results. In this work, we present the results of a detailed analysis procedure that was designed on the basis of previous works [1, 2, 3] in order to address the above questions. We will show that in fact, systematic and statistical effects can not explain the measured deviations between model expectations from QGSJET-II-2, QGSJET-II-04, EPOS 1.99 and SIBYLL 2.1 and the experimental measurement. The differences are observed at a significance level between $1.93 \sigma$ and $2.63 \sigma$, which implies a one-tailed C.L. from $0.43 \%$ to $2.68 \%$ that model predictions are statistically compatible with the experimental observation.

\section{The KASCADE-Grande array}

KASCADE-Grande (110 m a.s.l.) was an air-shower detector array designed to study cosmicray events with energies in the range of $10^{15}$ and $10^{18} \mathrm{eV}$ [9]. The detector was composed by several detectors systems aimed to measure with high precision distinct components and properties of the EAS, for example, the total number of charged particles (electrons plus muons) ( $E_{t h}>3 \mathrm{MeV}$ ) and muons $\left(E_{t h}>230 \mathrm{MeV}\right)$. The former, together with other gross shower variables like the EAS core position and the angle of incidence, were estimated from data collected by the $0.5 \mathrm{~km}^{2}$ main array of $37 \times 10 \mathrm{~m}^{2}$ scintillator detectors, while the latter, from local muon density measurements performed with the shielded detectors from the $200 \times 200 \mathrm{~m}^{2}$ KASCADE array (more details about the experiment can be found in $[9,10])$. At KASCADE-Grande systematic uncertainties for the core and the arrival direction of vertical EAS are found to be of the order of $6 \mathrm{~m}$ and $0.8^{\circ}$, respectively

\footnotetext{
* Speaker.
} 
[9]. On the other hand, for the total number of charged particles $\left(N_{c h}\right)$ the estimated resolution is $\leq 15 \%$ [9], while for the total number of muons $\left(N_{\mu}\right)$, it is $\leq 25 \%$ [11].

\section{Experimental and Monte Carlo data}

KASCADE-Grande was operated from December 2003 up to November 2012. The events analyzed in this work correspond to a data sample registered during the period December 2003 - October 2011. This data set is composed by selected events passing several instrumental and reconstruction cuts. The aim is to reduce systematic effects in the final results. For instance, to guarantee a data sample with stable detector performance only events taken during data acquisition runs and no hardware problems were accepted. Also data from runs without missing KASCADE detector clusters [10], and with more than 36 Grande working stations were considered. In addition, to exclude misreconstructed shower events affecting the quality of the data, just events that have passed successfully the full reconstruction chain [9] were included. On the other hand, low energy events, which have poorer reconstructed muon numbers, were removed from the data sample. This was achieved by requiring showers that activated more than 11 Grande stations and had a high $N_{\mu}$ number $\left(\gtrsim 4 \times 10^{4}\right)$. Besides, in order to avoid border effects and punch-through bias in the results, a cut on the location of the cores of the events was imposed. The EAS cores were required to be located within the limits of a central area of $8 \times 10^{4} \mathrm{~m}^{2}$ inside the KASCADE-Grande array. This constriction had also the advantage that it removed events from the analysis close to the center of the muon cluster and far way from it. The problem with these events is that after reconstruction they have over- and under-estimated muon numbers, respectively [11]. To end, in order to remove systematic effects due to a worsening of the pointing resolution of the detector at high zenith angles, showers with $\theta \geq 40^{\circ}$ were not included in the quality data sample. After applying selection cuts 2744850 events were left for the physical analysis and the corresponding total exposure was of about $1.3 \times 10^{13} \mathrm{~m}^{2} \cdot \mathrm{s} \cdot \mathrm{sr}$.

To compare the predictions of the hadronic interaction models to measured data, MC simulations were produced. First, CORSIKA [12] was used to simulate the production and development of the particle cascade. Then GEANT 3.21 [13] was employed to model the response of the detector to the passage of the shower. And finally, the standard KASCADE-Grande reconstruction algorithms were applied to reconstruct the MC events.

The U.S. standard atmosphere model as parameterized by Linsley was used for the MC simulations (see [12] and references therein). The choice of this model does not have an important influence over the present analysis, since the mean of the local RMS air pressure at the site is close to the magnitude predicted by the U.S. standard atmosphere model [14]. On the other hand, local variations of the air pressure and temperature were ignored in simulations, as they have a negligible influence on the shower muon content [14]. Furthermore, the curvature of the atmosphere was also neglected. This approximation is valid because the present analysis is limited to events with zenith angles $<40^{\circ}$, where a flat atmosphere model can be used [12].

The physics of the hadronic interactions was simulated using Fluka [15] at low energies $\left(E_{h} \leq\right.$ $200 \mathrm{GeV}$ ) combined with QGSJET-II-2, QGSJET-II-04, EPOS 1.99 and SIBYLL 2.1 as different alternatives to model the high-energy regime. For each of these models, different MC data samples were produced assuming distinct cosmic-ray composition scenarios: pure $\mathrm{H}, \mathrm{He}, \mathrm{C}, \mathrm{Si}$ and $\mathrm{Fe}$ 
nuclei, and a mixture of these elements in equal proportions. MC events were simulated for $\theta<42^{\circ}$ and for the energy interval $10^{15}-10^{18} \mathrm{eV}$ according to a $E^{\gamma}$ primary spectrum with spectral index of -2 . Data samples were later reweighted to produce additional data sets with $\gamma=-3.2,-3.0$ and -2.8 , which are closer to the measured one in the energy interval of interest [11].

MC Simulations were also useful to study the performance of the detector, to estimate systematic errors and to choose as well as to optimize the quality cuts above described [2]. The latter were also applied to the simulated data sets to avoid possible biases from the selection criteria on the conclusions of our study. After selection cuts the final number of simulated events for the five elemental nuclei are about $3.5 \times 10^{6}$ in case of QGSJET-II-2. For QGSJET-II-04, the number is $1.3 \times 10^{6}$, while for SIBYLL 2.1 and for EPOS 1.99 , it is around $1.2 \times 10^{6}$ in each case. The uncertainty analyses reveal that at high $N_{\mu}\left(>1.6 \times 10^{5}\right)$, the mean errors on the determination of the shower core position and the arrival zenith angle are $\lesssim 10 \mathrm{~m}$ and $\lesssim 0.6^{\circ}$, respectively, while the mean uncertainty for $N_{\mu}$ is $\lesssim 20 \%$. The latter decreases when increasing the size of the shower. Above $N_{\mu}>4.5 \times 10^{5}$, the mean uncertainties on the total muon number are smaller than $10 \%$. Full trigger and reconstruction efficiency of the KASCADE-Grande experiment was found above $\log _{10}(E / G e V)=7.00 \pm 0.20$ and $\log _{10} N_{\mu}=5.00 \pm 0.20$.

\section{The analysis method and the results}

The method is described in detail in references $[2,3]$. Here, we will outline briefly the main procedure. The principal goal of the study is to compare the measured zenith angle evolution of the muon data with the predictions of MC simulations in a model independent way. This is achieved by parameterizing the above evolution in terms of a muon attenuation length, $\Lambda_{\mu}$, which is estimated from data by means of the constant intensity cut method (CIC) [16]. Systematic and statistical errors are then carefully calculated. And finally the experimental value of $\Lambda_{\mu}$ is confronted with the estimations from the different hadronic interaction models, from which conclusions are drawn about the consistency of the model predictions with the measured value.

The analysis starts by correcting both experimental and simulated data for systematic errors using a correction function based on QGSJET-II-2 and parameterized in terms of the distance to the KASCADE center, the zenith of the EAS axis and the number of muons in the shower [2, 11]. The systematic uncertainties of the corrected $N_{\mu}$ become in this way independent of the core position, the zenith angle and the muon number with values within $10 \%[2,11]$. Next, the corrected data is divided in five zenith-angle intervals with equal aperture. Then, the integrated muon flux, $J\left(>N_{\mu}, \theta\right)$ (i.e. the number of showers detected above $N_{\mu}$ per unit solid angle, unit area and unit of time) for each of the above intervals is calculated (see fig. 1). To continue, five cuts at fixed energies or frequency rates are applied on the integral fluxes in the region of full efficiency and maximum statistics $\left(\log _{10} N_{\mu}=[5.2,6.0]\right)$ and from the intersections of each cut with the $J\left(>N_{\mu}, \theta\right)$ graphs, attenuation curves for the corrected $\log _{10} N_{\mu}$ in the atmosphere are extracted (c.f. fig. 1). The curves are parameterized as

$$
N_{\mu}(\theta)=N_{\mu}^{\circ} e^{-X_{0} \sec (\theta) / \Lambda_{\mu}},
$$

where $X_{0}=1022 \mathrm{~g} / \mathrm{cm}^{2}$ is the vertical column depth at KASCADE-Grande and $N_{\mu}^{\circ}$, a normalization factor, which is different for each attenuation curve. Finally, by applying a global fit to the curves, 

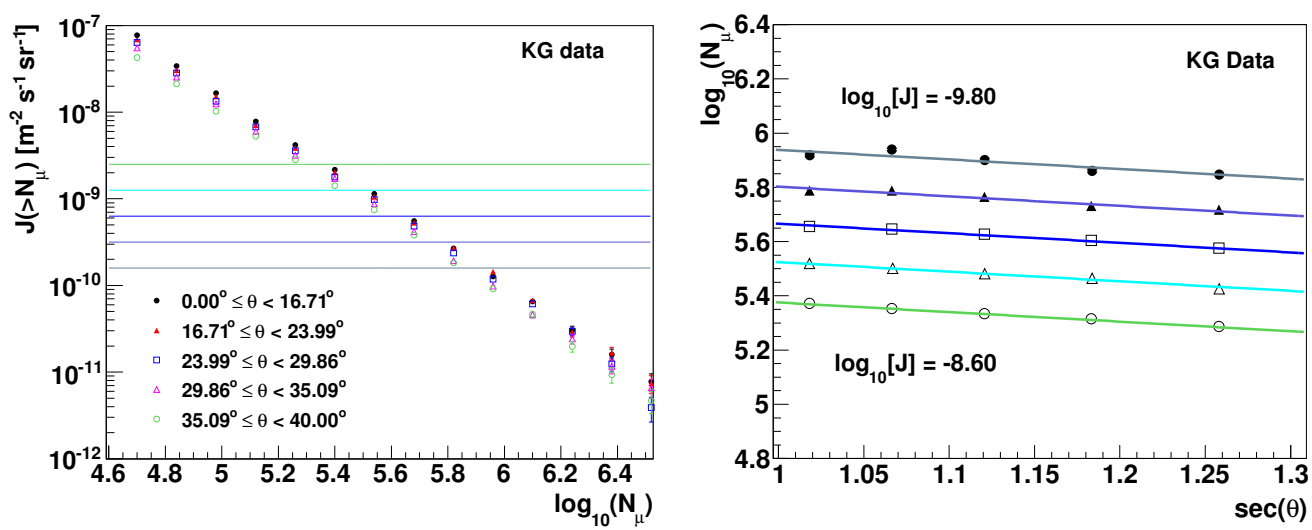

Figure 1: Left: Integral muon fluxes derived from the measurements with the KASCADE-Grande observatory for five zenith angle intervals: $\theta=\left[0^{\circ}, 16.7^{\circ}\right],\left[16.7^{\circ}, 24^{\circ}\right],\left[24^{\circ}, 29.9^{\circ}\right],\left[29.9^{\circ}, 35.1^{\circ}\right],\left[35.1^{\circ}, 40^{\circ}\right]$. The muon correction function was already applied to the data. The CIC cuts employed in this work are shown as horizontal lines. Right: Muon attenuation curves extracted with the CIC method from the experimental data. The results of the global fit with equation (4.1) is shown with solid lines. The highest curve correspond to the lowest CIC cut.

$\Lambda_{\mu}$ is obtained. A global fit was used, since the differences among the resulting $\Lambda_{\mu}$ values from individual fits to each attenuation curve are smaller than their respective uncertainties in the $N_{\mu}$ range under study.

The experimental result for $\Lambda_{\mu}$ is shown in table 1 along with the predictions from MC models for a mixed composition assumption. In the same table, the quoted errors represent the sum of the statistical and systematic uncertainties. Systematic errors were estimated through detailed studies. The corresponding results are displayed in table 2 .

The experimental systematic error includes the uncertainty due to the calculation procedure (construction of attenuation curves, global fit, number of zenith-angle intervals, position and number of CIC cuts, determination of attenuation curves, bin size), the uncertainty owing to the muon correction function and its model dependence, the systematic error of the corrected muon number and the uncertainties associated with the EAS core position relative to the center of the KASCADE muon array. The MC systematic error also involves uncertainties associated with the spectral index $(\gamma=-3.2,-2.8)$ and primary composition (using protons and iron nuclei as extreme composition assumptions). On the other hand, statistical errors are estimated by inducing random fluctuations on the number of events per $N_{\mu}$ interval and angular bin according to a Poisson distribution function.

Table 1: $\Lambda_{\mu}$ extracted from Monte Carlo and experimental data. Total uncertainties are also presented (systematic and statistical errors are added in quadrature).

\begin{tabular}{c|c|c|c|c|c}
\hline & QGSJET-II-2 & QGSJET-II-04 & EPOS 1.99 & SIBYLL 2.1 & KG data \\
\hline$\Lambda_{\mu}\left(\mathbf{g} / \mathbf{c m}^{2}\right)$ & $706_{-108}^{+87}$ & $735_{-145}^{+78}$ & $564_{-79}^{+49}$ & $743_{-205}^{+54}$ & $1256_{-258}^{+283}$ \\
\hline
\end{tabular}


Table 2: Systematic and statistical uncertainties (\%) on the predicted and experimental $\Lambda_{\mu}$.

\begin{tabular}{l|c|c|c|c|c}
\hline & QGSJET-II-2 & QGSJET-II-04 & EPOS 1.99 & SIBYLL 2.1 & KG data \\
\hline CIC method & $+9 /-1$ & $+8 /-2$ & $+8 /-1$ & $+4 /-9$ & $+9 /-2$ \\
EAS Core position & -3 & $+2 /-7$ & -3 & -7 & $+12 /-11$ \\
Muon systematics & +0.04 & -0.20 & +1 & -4 & $+13 /-10$ \\
Correction function & +7 & +1 & $+1 /-2$ & -3 & $+6 /-9$ \\
Statistical fluctuations & \pm 4 & \pm 3 & \pm 3 & \pm 4 & \pm 9 \\
Composition/spectral index & $+2 /-14$ & $+5 /-18$ & $3 /-13$ & $+4 /-24$ & - \\
Total & $+12 /-15$ & $+11 /-20$ & $+9 /-27$ & $+7 /-20$ & $+22 /-20$ \\
\hline
\end{tabular}

\section{Discussion}

The results from table 1 show that the muon attenuation length of EAS as measured with the KASCADE-Grande experiment is larger than the predictions from the high-energy hadronic interaction models. The significance of the deviations are presented in table 3 . They range from $+1.93 \sigma$ to $+2.63 \sigma$. This means that the probability of agreement (calculated as the one-tailed C.L.) between the experiment and the models is low. The table 3 show us that this probability is just between $0.43 \%$ and $2.68 \%$, which implies that the measured value of $\Lambda_{\mu}$ is not statistically consistent with the predictions of the high-energy hadronic interaction models here studied.

The influence on the results of additional sources of systematic uncertainties were also analysed. Instrumental effects like the aging of the detector were discarted. In the latter, for example, no important effect was observed (variations are within $\pm 0.14 \sigma$ for data samples corresponding to three different periods each with an effective time of $\approx 10^{7} \mathrm{~s}$ ). On the other hand, the measured statistical fluctuations of the muon densities, $\rho_{\mu}(r)$, are different from the ones predicted from MC simulations. This introduces an additional systematic error on the MC expectations. However, this error $(+15 \%)$ was not enough to explain the observed discrepancy. Errors due to systematics on the EAS core location, the reconstruction of the arrival direction and the $\rho_{\mu}(r)$ distributions were also studied with MC simulations and they were found to be within $\pm 3 \%$. Differences in the shape of the lateral distribution function of muons for experimental and MC data were also studied and the results did not show and important influence on $\Lambda_{\mu}$.

Further studies [2] have allowed to track down the anomaly to the behavior of the radial muon density distributions in the atmosphere, which are free of systematics from the muon correction function, the CIC method, etc. These distributions in addition are the basis for the reconstruction of the muon number of the EAS [9]. The analysis have shown that the measured $\rho_{\mu}(r)$ distributions of EAS decrease with the zenith angle more slowly than in MC simulations as it was the case for

Table 3: Significance of the differences between the measured $\Lambda_{\mu}$ and the MC expectations. The probabilities (one-tailed C.L.) of agreement between experiment and models are also presented.

\begin{tabular}{l|c|c|c|c}
\hline & QGSJET-II-2 & QGSJET-II-04 & EPOS 1.99 & SIBYLL 2.1 \\
\hline Deviation $(\sigma)$ & +2.02 & +1.93 & +2.63 & +1.94 \\
C.L. $(\%)$ & 2.17 & 2.68 & 0.43 & 2.62 \\
\hline
\end{tabular}




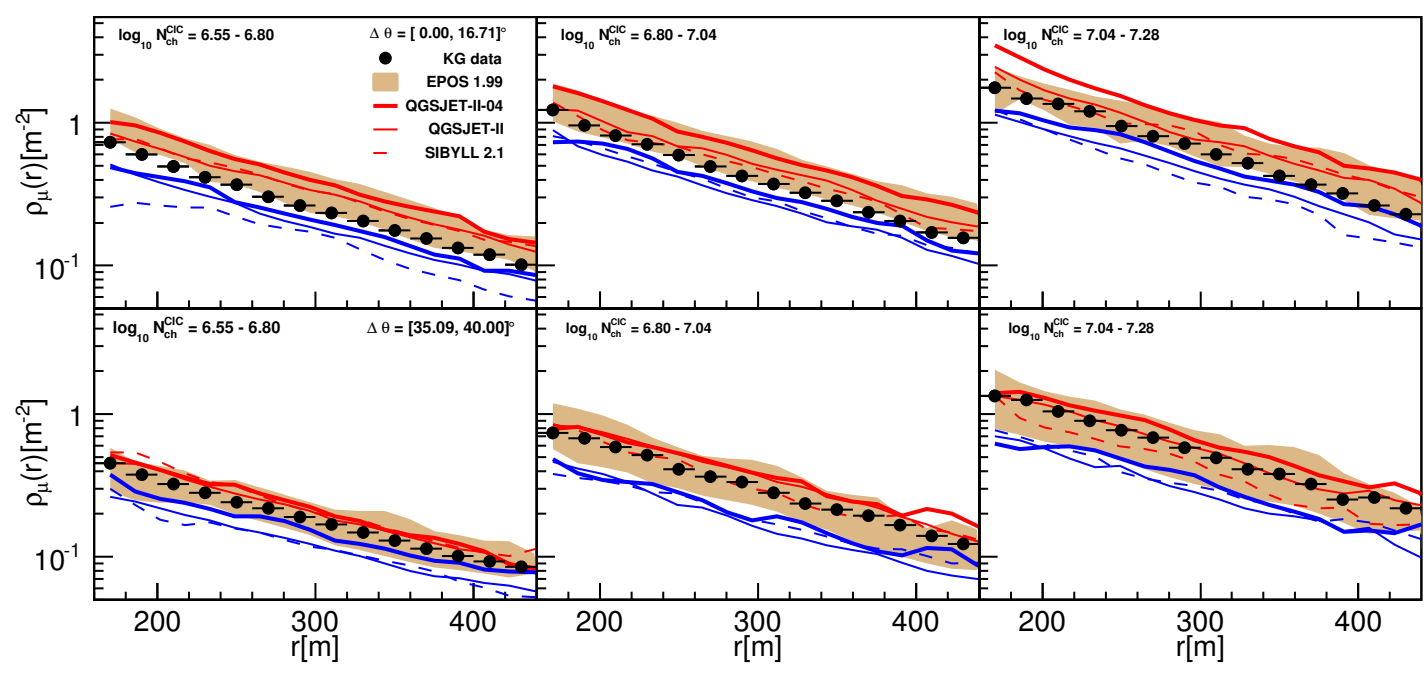

Figure 2: Measured radial muon density distributions (points) compared with the predictions of MC simulations (bands and lines). The red (upper) lines are the predictions for a heavy ( $\mathrm{Si}+\mathrm{Fe})$ composition scenario, while the blue (lower) curves correspond to the expectations for a light $(\mathrm{H}+\mathrm{He})$ composition assumption. Data is presented for two different zenith angle intervals: $\theta=\left[0^{\circ}, 16.7^{\circ}\right]$ (upper panels) and $\left[35.1^{\circ}, 40^{\circ}\right]$ (lower panels), and three distinct $\log _{10} N_{c h}^{C I C}$ ranges (from left to right): [6.55, 6.80], [6.80, 7.04], [7.04, 7.28].

the $N_{\mu}$ integral fluxes (see also [2]).

For the above study, data for each zenith angle in the range of full efficiency and maximum statistics was further divided in three $\log _{10} N_{c h}^{C I C}$ intervals, where $N_{c h}^{C I C}$ is the number of charged particles that a shower could have at $\theta=22^{\circ}$. It would be desirable to divide the data in energy intervals, but since the energy of the measured showers is less precisely known we use instead $N_{c h}^{C I C}$. This observable was calculated event-by-event using the CIC method as described in [3]. According to the CIC method, all events with the same $N_{c h}^{C I C}$ at different zenith angles should have the same mean energy. What we obtained then is a picture of the zenith angle evolution of $\rho_{\mu}(r)$ at three different energy intervals, as shown in fig. 2. In this figure the experimental muon distributions are compared with the predictions from MC simulations for a light $(\mathrm{H}+\mathrm{He})$ and a heavy $(\mathrm{Si}+\mathrm{Fe})$ composition. Clearly, it is seen that the experimental and simulated $\rho_{\mu}(r)$ distributions evolve with the zenith angle in a different way. In particular, the expected radial muon density distributions from simulations change faster than the measured ones. This effect seems to be more remarkable at higher energies for inclined showers $\left(35.1^{\circ} \leq \theta \leq 40^{\circ}\right)$, where the muon measurements are observed to stand above the predictions obtained with SIBYLL 2.1. Even more, for the same zenith angle interval, the measurements are located at the upper limit of the QGSJETII-2 predictions. This effect may be connected with the muon excess measured at the Pierre Auger observatory at higher energies, which also increases with the zenith angle [17].

\section{Conclusions}

The muon attenuation length of EAS was measured at KASCADE-Grande in the energy interval from $10^{16.3}$ to $10^{17.3} \mathrm{eV}$ and compared with the predictions from the high-energy hadronic in- 
teraction models QGSJET-II-2, QGSJET-II-04, EPOS 1.99 and SIBYLL 2.1. The results show that the measured value deviates by $+1.93 \sigma$ to $+2.63 \sigma$ from the predictions of the models. This implies that the zenith angle evolution of the muon content of showers as observed at the KASCADEGrande altitude is not statistically consistent with predictions from the above models. The statistical analysis shows that the probability of agreement between model and experiment ranges from $0.43 \%$ to $2.68 \%$. Statistical and systematic errors (associated to instrumental effects, reconstruction/analysis methods, EAS fluctuations, etc.) can not explain the observed anomaly. The latter is due to the fact that the $\rho_{\mu}(r)$ distributions of the experimental data evolve more slowly with the zenith angle than the simulated ones. The difference seems to be more important at high-energies and for inclined shower $\left(35.1^{\circ} \leq \theta \leq 40^{\circ}\right)$. In this regime, the expectations from SIBYLL 2.1 for the $\rho_{\mu}(r)$ distributions of the heavy/light nuclei do not bracket the experimental one, as the model predicts a lower number of muons than observed.

Acknowledgment: The authors would like to thank the members of the engineering and technical staff of the KASCADE-Grande collaboration, who contributed to the success of the experiment. The KASCADE-Grande experiment is supported in Germany by the BMBF and by the "Helmholtz Alliance for Astroparticle Physics - HAP" funded by the Initiative and Networking Fund of the Helmholtz Association, by the MIUR and INAF of Italy, the Polish Ministry of Science and Higher Education, and the Romanian Authority for Scientific Research UEFISCDI (PNII-IDEI grants 271/2011 and 17/2011). J.C.A.V. acknowledges the partial support of CONACyT and the Proalmex grant (2015).

\section{References}

[1] J.C. Arteaga-Velazquez et al., EPJ Web of Conferences 52 (2013) 07002.

[2] J.C. Arteaga-Velazquez et al., Proc. of the 33rd ICRC, Rio de Janeiro, Brazil, icrc772 (2013).

[3] J.C. Arteaga-Velazquez et al., Proc. of the 18th ISVHECRI, CERN, to be published.

[4] S.S. Ostapchenko, Phys. Rev. D 83 (2011) 014018.

[5] T. Pierog et al., arxiv: 1306.0121 (2013).

[6] S.S. Ostapchenko, Nucl. Phys. B (Proc. Suppl.) 151 (2006) 143; S. Ostapchenko, Phys. Rev. D 74 (2006) 014026.

[7] T. Pierog et al., Report FZKA 7516, Germany (2009) 133.

[8] E.J. Ahn et al., Phys. Rev D 80 (2009) 094003.

[9] Apel W. D. et al., NIM A $\mathbf{6 2 0}$ (2010) 202.

[10] Antoni T. et al., NIM A $\mathbf{5 1 3}$ (2003) 490.

[11] Apel W. D. et al., Astrop. Phys. 36 (2012) 183.

[12] D. Heck et al., Report FZKA 6019, Forschungszentrum Karlsruhe (1998).

[13] R. Brun, F. Carminati, CERN Program Library Long Writeup (1993) W5013.

[14] Daniel Fuhrmann, KASCADE-Grande measurements of energy spectra for elemental groups of cosmic rays, Ph.D. thesis, University of Wuppertal, Germany (2012).

[15] A. Fassò et al., Report CERN-2005-10, SLAC-R-773 (2005).

[16] J. Hersil et al., Phys. Rev. Lett. 6 (1961) 22; D. M. Edge et al., J. Phys. A 6 (1973) 1612.

[17] P. Abreu et al., arXiv:1107.4809 (2011); A. Yushkov et al., Eur. Phys. J. Web. Conf. 53 (2013) 07002; L. Nellen et al., J. Phys.: Conf. Ser. 409 (2013) 012107. 